\title{
Intraoperative Fluoroscopy Improves Recreation of Femoral Offset but not Leg Lengths during Direct Anterior Total Hip Arthroplasty
}

\author{
Evan J Smith, $M D^{1}$, George Ibrahim, $M D^{1}$, Ryan D Scully, $M D^{2 *}$, Gautam Siram, $M D^{3,4}$, Anthony $S$ \\ Unger, $M D^{4,5}$ and J Stuart Melvin, $M D^{4,5}$
}

${ }^{1}$ George Washington University, Washington, USA

${ }^{2}$ United States Naval Hospital Yokosuka, Japan

${ }^{3}$ Summit Orthopedics, Washington, USA

${ }^{4}$ The Gildenhorn Institute of Bone and Joint Health, Washington, USA

${ }^{5}$ Washington Orthopaedics and Sports Medicine, Washington, USA

\begin{abstract}
Background: Postoperative leg length discrepancy (LLD) and increased femoral offset (FO) after total hip arthroplasty (THA) are frequently cited reasons for decreased patient satisfaction and litigation. Minimizing LLD and recreating FO can be challenging and different approaches exist. Some surgeons perform total hip arthroplasty from a direct anterior approach (DAA) utilizing intraoperative fluoroscopyto minimize potential for LLD and changes in FO. Others utilizing the DAAdo not use fluoroscopy but rely on direct intraoperative clinical comparison.
\end{abstract}

Methods: We retrospectively evaluated differences in postoperative LLD and FO between DAA THA cases performed with and without the use of intraoperative fluoroscopy.

Results: The use of fluoroscopy did not appear to provide increased accuracy or precision in avoiding postoperative LLD compared to clinical comparison techniques. However, there was a significant difference noted in the ability to reestablish FO. The absence of fluoroscopy resulted in a mean increase to native FO $(4.7 \mathrm{~mm}$ vs $0.1 \mathrm{~mm})$ and more frequent outliers.

Conclusion: The use of fluoroscopy during DAA THA does not provide significant clinical benefit with regard to minimizing postoperative LLD. Fluoroscopy may help in normalizing FO, as this is difficult to evaluate without imaging.

Keywords

Total hip arthroplasty, Femoral offset, Limb length discrepancy, Direct anterior approach

\section{Introduction}

A change in leg lengths (LL) after total hip arthroplasty (THA) is a frequently cited reason for decreased patient satisfaction and litigation [1]. Patients may notice a leg length discrepancy (LLD) as little as $5 \mathrm{~mm}$ [2]. Increased leg lengths can cause chronic back pain, sciatic nerve dysfunction, and the need for heel lifts. Abductor weakness and hip instability can result from decreased leg lengths [2-4].

Similarly, changes to native femoral offset may lead to patient dissatisfaction. Increases in offset as little as $5 \mathrm{~mm}$ may result in decreased outcome scores, hip pain, and abductor dysfunction $[5,6]$. Decreases in offset result in decreased abductor tension, impingement, and increased joint reactive forces [7]. Failure to closely restore the native femoral offset can also lead to accelerated polyethylene wear and aseptic loosening [8-10].

Arthroplasty performed through a direct anterior approach (DAA) in the supine position allows for use of intra-operative fluoroscopy, which can accommodate for intra-operative changes in pel- vic position and femoral rotation. Intra-operative fluoroscopy may improve implant positioning and precision leading to more accurate recreation of native LL and FO. However, many surgeons perform anterior THA without fluoroscopy and achieve excellent results, claiming the supine positioning allows direct comparison to the contralateral leg $[11,12]$. This study aims to compare the ability to recreate native $L L$ and FO offset between a surgeon who performs DAA

*Corresponding author: Ryan D Scully, MD, United States Naval Hospital Yokosuka, Japan

Accepted: May 09, 2020

Published online: May 11, 2020

Citation: Smith EJ, Ibrahim G, Scully RD, et al. (2020) Intraoperative Fluoroscopy Improves Recreation of Femoral Offset but not Leg Lengths during Direct Anterior Total Hip Arthroplasty. J Orthop Surg Tech 3(1):115-119 
Citation: Smith EJ, Ibrahim G, Scully RD, et al. (2020) Intraoperative Fluoroscopy Improves Recreation of Femoral Offset but not Leg Lengths during Direct Anterior Total Hip Arthroplasty. J Orthop Surg Tech 3(1):115-119

THA with fluoroscopic assistance and another who performs DAA THA with direct limb comparison without fluoroscopy. Additionally, we aim evaluate whether patient factors contribute to imprecision with either technique.

\section{Materials and Methods}

After receiving Institutional Review Board approval, we performed a retrospective review of the medical records of 200 patients with hip osteoarthritis who underwent DAA THA. 100 consecutive patients for two high-volume, fellowship-trained arthroplasty surgeons who routinely utilize the DAA for THA were included beginning January 1, 2016. Surgeon A performed DAA THA utilizing a specialized table (Hana ${ }^{\circledR}$, Mizuho OSI) and intra-operative fluoroscopy. Surgeon B performed DAA THA on a conventional operating table, without the use of intra-operative fluoroscopy, with the contralateral lower extremity free for clinical comparison to assess range of motion, limb length, and offset tension. Eighty-two patients (40 from Surgeon A, 42 from Surgeon B) were excluded due to incomplete clinical data, inadequate radio graphs significant deformity, or contralateral hip replacement. Overall, 118 patients were included. A priori power analysis revealed that 51 and 48 patients were required to detect clinically significant differences in LLD and FO of $5 \mathrm{~mm}$,

Table 1: Comparison of demographic data between study groups.

\begin{tabular}{|l|l|l|l|}
\hline & No imaging & Imaging & p value \\
\hline Pts & 58 & 60 & \\
\hline M/F & $17 / 41$ & $24 / 36$ & 0.22 \\
\hline Left/Right & $26 / 32$ & $27 / 33$ & \\
\hline Mean Age & 68.62 & 63.90 & 0.01 \\
\hline Range & $49-84$ & $23-91$ & \\
\hline Mean BMI & 26.31 & 26.90 & 0.10 \\
\hline BMI > 30 & $9(16 \%)$ & $19(32 \%)$ & 0.04 \\
\hline Head Size 28/32/36/40 & $2 / 36 / 20 / 0$ & $5 / 15 / 34 / 6$ & \\
\hline
\end{tabular}

respectively, with an alpha of 0.05 and a power of 0.8 . Demographic data is summarized in (Table 1 ).

A single preoperative and three postoperative low anteroposterior (AP) pelvis radiographs were reviewed by 2 independent orthopaedic surgeons. The mean LLD and FO of each patient were used for statistical analysis. LL measurements were performed by first creating an inter-teardrop line. The length of a vertical line from the inter-teardrop line to the most prominent point on the lesser trochanter determined the side-specific LL (Figure 1). LL measurements for the operative side were compared to the nonoperative side to determine leg length discrepancy (LLD). FO measurements were performed to measure the global offset of the femur. A line was extended down the longaxis of the femur. A second line extending from the center of rotation of the femoral head to the medial wall of the acetabulum was the measurement of FO (Figure 1). Images were calibrated to a $25.4 \mathrm{~mm}$ marker ball. Postoperative images were calibrated to the known size of the femoral head implants [13-15]. Changes in FO were determined by comparing preoperative and postoperative radiographs. Student's t-test was used to compare means and chi-square analysis was performed to compare the percentage of subgroup frequency.

\section{Results}

There were small variations in demographics amongst the groups. The group using fluoroscopy was significantly younger $(68.6$ vs 63.9) and had a significantly higher number of patients with $\mathrm{BMI}>$ 30 ( $16 \%$ vs $32 \%$, (Table 1 ). Additionally, $36 \mathrm{~mm}$ heads were more frequently used in cases with imaging whereas cases not using imaging most frequently used $32 \mathrm{~mm}$ heads. Both groups excluded similar numbers with the most frequent reason being incomplete postoperative imaging (Figure 1, Figure 2, Table 1, Table 2 and Table 3).

There was a small but statistically significant difference between surgeons in postoperative LLD measurements. Preoperative LLD measurements were comparable between the groups (Table 2). The use of intraoperative imaging resulted in a mean $1.4 \mathrm{~mm} \mathrm{LL}$ increase while lack of fluoroscopy resulted in a mean decrease of $0.6 \mathrm{~mm}$. Both modalities were successful at normalizing postoperative LL. Ad-

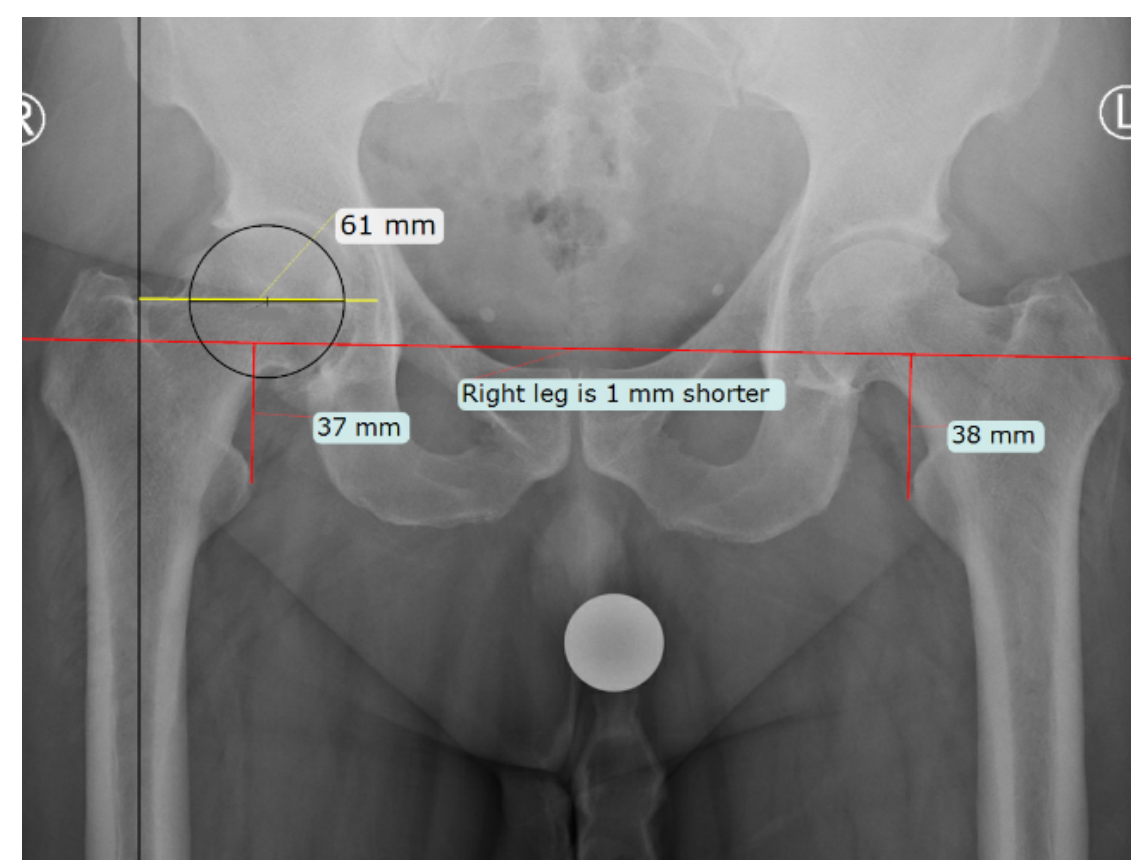

Figure 1: Standing low AP pelvis radiograph demonstrating methods of measuring FO (yellow line), LL (red vertical lines). The horizontal red line represents the inter-teardrop line. 
Citation: Smith EJ, Ibrahim G, Scully RD, et al. (2020) Intraoperative Fluoroscopy Improves Recreation of Femoral Offset but not Leg Lengths during Direct Anterior Total Hip Arthroplasty. J Orthop Surg Tech 3(1):115-119

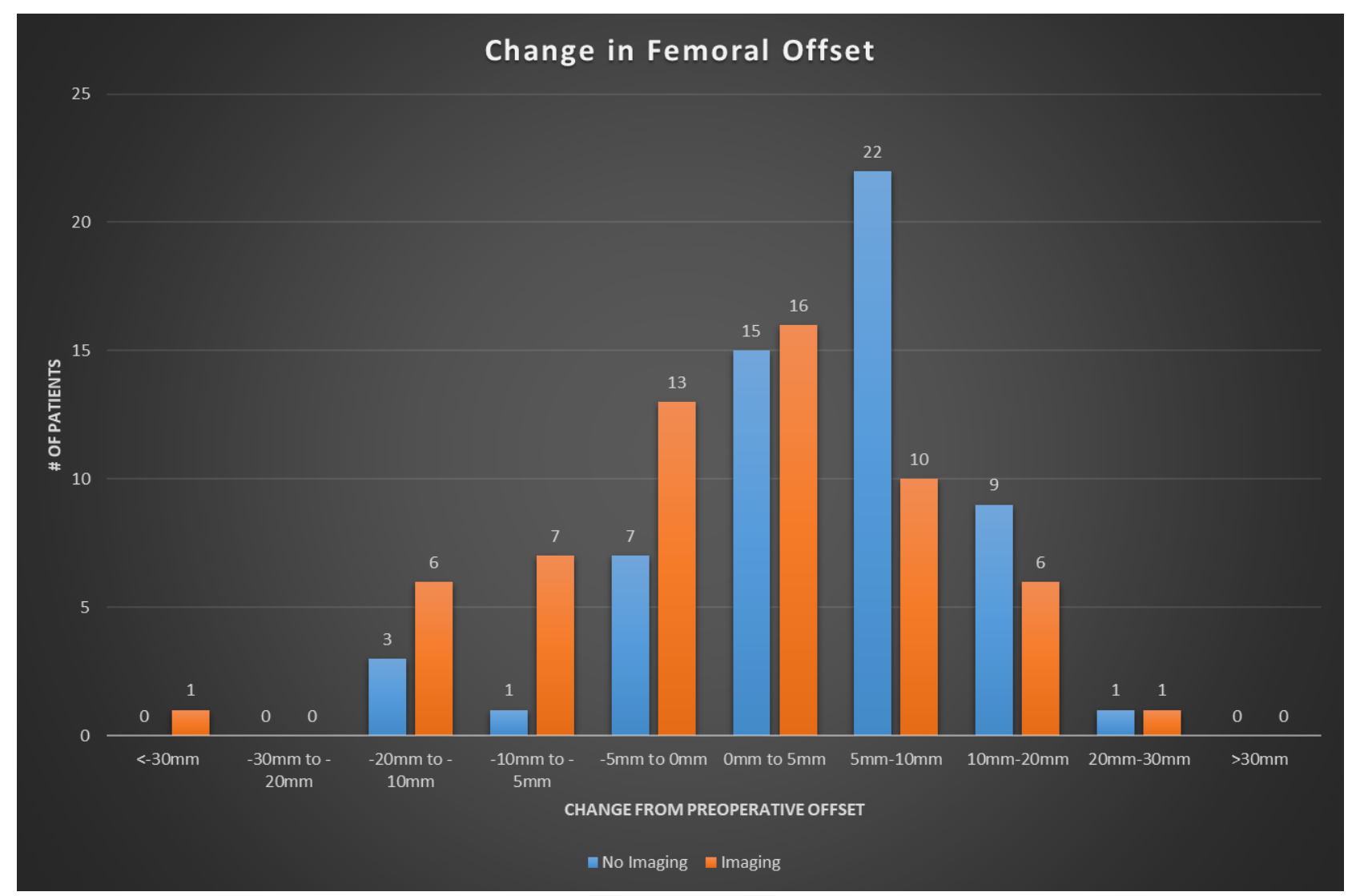

Figure 2: Comparison of postoperative change in femoral offset, subgroup totals.

Table 2: Comparison of preoperative (left) and postoperative (right) LLD between study groups, including mean and subgroup differences.

\begin{tabular}{|l|l|l|l|l|l|l|l|}
\hline Preoperative & No imaging & Imaging & p value & Postoperative & No imaging & Imaging & p value \\
\hline Mean $(\mathrm{mm})$ & -4.18 & -3.43 & 0.51 & Mean $(\mathrm{mm})$ & -0.6 & 1.4 & 0.01 \\
\hline$<-10 \mathrm{~mm}$ & 8 & 4 & 0.20 & $<-10 \mathrm{~mm}$ & 2 & 0 & 0.15 \\
\hline$<-5 \mathrm{~mm}$ & 20 & 25 & 0.42 & $<-5 \mathrm{~mm}$ & 8 & 3 & 0.10 \\
\hline$>5 \mathrm{~mm}$ & 2 & 2 & 0.97 & $>5 \mathrm{~mm}$ & 0 & 10 & 2 \\
\hline$>10 \mathrm{~mm}$ & 0 & 0 & 0.00 & $>10 \mathrm{~mm}$ & 0.48 & 0.16 \\
\hline Within $5 \mathrm{~mm}$ of epual & 36 & 33 & 0.44 & Within $5 \mathrm{~mm}$ of epual & 43 & 47 & 0.59 \\
\hline
\end{tabular}

Table 3: Comparison of postoperative change in femoral offset, mean and sub group differences.

\begin{tabular}{|l|l|l|l|}
\hline & No imaging & Imaging & p value \\
\hline Mean $(\mathrm{mm})$ & 4.71 & 0.1 & $\mathbf{0 . 0 0 3}$ \\
\hline$<-30 \mathrm{~mm}$ & 0 & 1 & 0.32 \\
\hline$-30 \mathrm{~mm}$ to $-20 \mathrm{~mm}$ & 0 & 0 & N/A \\
\hline$-20 \mathrm{~mm}$ to $-10 \mathrm{~mm}$ & 3 & 6 & 0.32 \\
\hline$-10 \mathrm{~mm}$ to $-5 \mathrm{~mm}$ & 1 & 7 & $\mathbf{0 . 0 2}$ \\
\hline$-5 \mathrm{~mm}$ to $0 \mathrm{~mm}$ & 7 & 13 & 0.16 \\
\hline $0 \mathrm{~mm}$ to $5 \mathrm{~mm}$ & 15 & 16 & 0.92 \\
\hline $5 \mathrm{~mm}-10 \mathrm{~mm}$ & 22 & 10 & 0.009 \\
\hline $10 \mathrm{~mm}-20 \mathrm{~mm}$ & 9 & 6 & 0.37 \\
\hline $20 \mathrm{~mm}-30 \mathrm{~mm}$ & 1 & 1 & 0.98 \\
\hline$>30 \mathrm{~mm}$ & 0 & 0 & N/A \\
\hline
\end{tabular}

ditionally, both techniques resulted in a high proportion of patients within $5 \mathrm{~mm}$ of the contralateral side (Table 2) without significant differences.

Using intraoperative imaging resulted in more accurate postoperative femoral offset. Lack of fluoroscopy resulted in a mean offset increase of $4.7 \mathrm{~mm}$ compared to a mean increase of $0.1 \mathrm{~mm}$ when using imaging (Table 3 ) and resulted in significantly more patients with offset increased between $5 \mathrm{~mm}$ and $10 \mathrm{~mm}$. Both groups had a similar number of outliers; there was no statistically significant difference in the number of patients with increased or decreased offset greater than $10 \mathrm{~mm}$. In the non-fluoroscopy group, $75 \%$ of patients were within $10 \mathrm{~mm}$ of the preoperative offset, and $38 \%$ were within $5 \mathrm{~mm}$ of the preoperative offset.Using fluoroscopy resulted in $77 \%$ of patients within $10 \mathrm{~mm}$ of preoperative offset and $48 \%$ within 5 $\mathrm{mm}$.

\section{Discussion}

The use of intraoperative fluoroscopy during THA has been popularized with increased use of the DAA. Supine positioning facilitates 
Citation: Smith EJ, Ibrahim G, Scully RD, et al. (2020) Intraoperative Fluoroscopy Improves Recreation of Femoral Offset but not Leg Lengths during Direct Anterior Total Hip Arthroplasty. J Orthop Surg Tech 3(1):115-119

use of real-time imaging to assess implant positioning and alignment without limiting access to the surgical field. Proponents for the use of fluoroscopy report improved cup positioning and normalization of native femoro-pelvic relationships. While several studies have looked at the cup positioning and found reliable results with and without imaging, few have studied the impact of using fluoroscopy on normalization of leg length and femoral offset for DAA THA $[11,12,16-18]$. To our knowledge, this is the first study evaluating the utility of intraoperative fluoroscopy when attempting to normalize $\mathrm{LL}$ and FO during DAA THA.

Our results indicate there was a difference in LLD between the different techniques. However, both surgeons were within $2 \mathrm{~mm}$ of recreating the contralateral limb length and this difference was not clinically significant. Postoperative LLD of as little as $5 \mathrm{~mm}$ have been reported to be clinically significant [2], however, most reports have noted LLD of $10 \mathrm{~mm}$ or greater to be clinically relevant [19-22]. We report only 2 patients ( $3 \%$ ) in each group with postoperative LLD $>10 \mathrm{~mm}$. Using fluoroscopy, other groups have published similar findings reporting rates of postoperative LLD > $10 \mathrm{~mm}$ from $0-10 \%$ $[16,18,23-25]$. Fewer studies have reported on radiographic parameters after DAA THA without fluoroscopy: Tian, et al. reported a mean LLD of $2 \mathrm{~mm}$ postoperatively [12]. Bigham et al. reported a mean discrepancy of $0.8 \mathrm{~mm}[17]$ of these two studies, neither reported on outliers and precision. We report excellent accuracy and precision without the use of fluoroscopy.

Despite less clinical focus compared to LLD, even small changes in FO may negatively affect patient outcomes. While inconsistent, previous reports on the effect of offset on outcomes has shown increases of as little as $5 \mathrm{~mm}$ may lead to abductor pain and decreased patient satisfaction $[6,7]$. Decreases in offset affect the abductor lever arm and may result in increased joint reactive forces and accelerated implant wear [8,26-29]. Significant decrease in offset may result in instability and dislocation $[8,29]$. Tian, et al. recently reported on a cohort of 1017 THAs performed without fluoroscopy noting a postoperative offset within $4 \mathrm{~mm}$ in $90 \%$ of cases [12]. Using fluoroscopy, Lin, et al reported acceptable postoperative offset (within $5 \mathrm{~mm}$ ) in $75 \%$ of patients [30]. However, both studies used a FO measurement from the center of rotation of the femoral head. This method does not accurately reflect abductor tension since medializing the cup during implantation may recreate FO but reduce abductor tension. We employed a FO measurement method that evaluates global offset. Since the abductors originate on the pelvis and insert onto the femur, a measurement that includes the pelvis and the femur is necessary to thoroughly understand changes in abductor length. Using a similar global offset measurement, Austin, et al. reported FO within $5 \mathrm{~mm}$ in $58 \%$ of hips using fluoroscopy and $89 \%$ within $10 \mathrm{~mm}$ [18]. With similar findings, we report $48 \%$ of patients with in $5 \mathrm{~mm}$ of preoperative offset and $77 \%$ within $10 \mathrm{~mm}$. in our series, lack of fluoroscopy, led to $38 \%$ of patients within $5 \mathrm{~mm}$ of preoperative offset and $75 \%$ with in $10 \mathrm{~mm}$.

We found increased accuracy in recreating preoperative FO when using fluoroscopy. Lack of fluoroscopy resulted in an increase in mean postoperative FO $(4.7 \mathrm{~mm}$ vs $0.1 \mathrm{~mm})$ and specifically resulted in an increased percentage of patients with offset increased between $5-10 \mathrm{~mm}$ (38\% vs $17 \%)$. Fluoroscopy may have afforded improved FO accuracy by allowing of direct fluoroscopic comparison to pre-arthroplasty images to recreate preoperative offset as well as comparison to the contralateral hip.

There are several limitations to the current study. CT imaging is the most reliable means for evaluating changes in LL and FO [31]. Radiographic imaging is inconsistent with regards to pelvic rotation, tilt and femoral flexion and abduction. All these changes can affect measurements of $\mathrm{LL}$ and FO. Relying on a single pelvic image can misrepresent true measurements; therefore, an attempt was made to mitigate this problem by including three postoperative measurements. Nevertheless, we were limited by having only one preoperative image for most patients, which is common for patients undergoing THA. Additionally, variability exists between and within evaluators. While we did not perform an analysis of reliability, our measurement techniques have well-established high rates of inter- and intra-observer reliability [13-15]. While both surgeons approached THA with the aim to restore $\mathrm{FO}$, individual stability and nuance within cases becomes lost when reporting in a retrospective fashion. Additionally, surgeon preference for varying head sizes may have affected group differences in FO (increased offset noted in group using $32 \mathrm{~mm}$ heads.) Similarly, differences in femoral stem choice may affect FO. We believe this had little if any effect on our results due to the similar offset across standard sizes for the implants used by each surgeon.

The question of whether fluoroscopy provides value in THA is complex and may vary based on practice setting, geographical location, surgeon experience, patient population, and multiple financial variables. This is the first study comparing DAA THA with and without fluoroscopy to determine the effect on postoperative $L L$ and FO. Previous studies have reported reliable cup positioning with and without the use of fluoroscopy with low dislocation rates [11,12]. Our study as well as other contemporary studies provide evidence that the use of fluoroscopy does not provide significant benefit with respect to normalizing leg length $[12,16-18]$. We report here that differences may exist in offset normalization. While relatively under-studied, changes in FO may result in variation in implant longevity as well as patient reported outcomes. Further research is warranted to investigate the equipment and OR costs incurred from the use of fluoroscopy to fully characterize the value of intraoperative fluoroscopy.

\section{Conclusion}

The use of fluoroscopy during DAA THA does not appear to provide significant clinical benefit with regard to minimizing postoperative LLD compared to direct manual comparison without fluoroscopy. However, fluoroscopy may benefit the surgeon in regards to normalizing FO as this is difficult to evaluate without imaging. Increased femoral offset may result in increased abductor tension, lateral hip discomfort and decreased patient outcomes.

\section{References}

1. Hofmann AA, Skrzynski MC (2000) Hip arthroplasty: Headaches \& migraines: Leg-length inequality and nerve palsy in total hip arthroplasty: A Lawyer A waits. Orthopedics 23: 943-944.

2. Edeen J, Sharkey PF, Alexander AH (1995) Clinical significance of leg-length inequality after total hip arthroplasty. Am J Orthop (Belle Mead NJ) 24: 347-351.

3. Konyves A, Bannister GC (2005) The importance of leg length discrepancy after total hip arthroplasty. J Bone Joint Surg Br 87: 155-157.

4. Wylde V, Whitehouse SL, Taylor AH, et al. (2009) Prevalence and functional impact of patient-perceived leg length discrepancy after hip replacement. Int Orthop 33: 905-909.

5. Incavo SJ, Havener T, Benson E, et al. (2004) Efforts to improve cementless femoral stems in THR: 2-to 5-year follow-up of a high-offset femoral stem with distal stem modification (Secur-Fit Plus). J Arthroplasty 19: 61-67.

6. Liebs TR, Nasser L, Herzberg W, et al. (2014) The influence of femoral offset on health-related quality of life after total hip replacement. Bone Joint J 96: 36-42. 
Citation: Smith EJ, Ibrahim G, Scully RD, et al. (2020) Intraoperative Fluoroscopy Improves Recreation of Femoral Offset but not Leg Lengths during Direct Anterior Total Hip Arthroplasty. J Orthop Surg Tech 3(1):115-119

7. McGrory BJ, Morrey BF, Cahalan TD, et al. (1995) Effect of femoral offset on range of motion and abductor muscle strength after total hip arthroplasty. J Bone Joint Surg Br 77: 865-869.

8. Sakalkale DP, Sharkey PF, Eng K, et al. (2001) Effect of femoral component offset on polyethylene wear in total hip arthroplasty. Clin Orthop 388: 125-134.

9. Little NJ, Busch CA, Gallagher JA, et al. (2009) Acetabular polyethylene wear and acetabular inclination and femoral offset. Clin Orthop 467: 2895-2900.

10. Hodge WA, Andriacchi TP, Galante JO (1991) A relationship between stem orientation and function following total hip arthroplasty. J Arthroplasty 6: 229-235.

11. Soderquist MC, Scully R, Unger AS (2017) Acetabular placement accuracy with the direct anterior approach freehand technique. J Arthroplasty 32: 2748-2754.

12. Tian S, Goswami K, Manrique J, Blevins K, et al. (2019) Direct anterior approach total hip arthroplasty using a morphometrically optimized femoral stem, a conventional operating table, without fluoroscopy. The Journal of arthroplasty 34: 327-332.

13. Meermans G, Malik A, Witt J, et al. (2011) Preoperative radiographic assessment of limb-length discrepancy in total hip arthroplasty. Clin Orthop 469: 1677-1682.

14. Kjellberg M, Al Amiry B, Englund E, et al. (2012) Measurement of leg length discrepancy after total hip arthroplasty. The reliability of a plain radiographic method compared to CT-scanogram. Skeletal Radiol 41: 187-191.

15. Ellapparadja P, Mahajan V, Deakin AH, et al. (2015) Reproduction of hip offset and leg length in navigated total hip arthroplasty: How accurate are we? J Arthroplasty 30: 1002-1007.

16. Nam D, Sculco PK, Abdel MP, et al. (2013) Leg-length inequalities following THA based on surgical technique. Orthopedics 36 e395-e400.

17. Bingham JS, Spangehl MJ, Hines JT, et al. (2018) Does intraoperative fluoroscopy improve limb-length discrepancy and acetabular component positioning during direct anterior total hip arthroplasty? The Journal of arthroplasty 33: 2927-2931.

18. Austin MS, Rothman RH (2009) Acetabular orientation: anterolateral approach in the supine position. Clinical orthopaedics and related research 467: 112-118.

19. Desai AS, Dramis A, Board TN (2013) Leg length discrepancy after total hip arthroplasty: A review of literature. Curr Rev Musculoskelet Med 6: 336-341.
20. Whitehouse MR, Stefanovich-Lawbuary NS, Brunton LR, et al. (2013) The impact of leg length discrepancy on patient satisfaction and functional outcome following total hip arthroplasty. J Arthroplasty 28: 1408-1414.

21. Keršič M, Dolinar D, Antolič V, et al. (2013) The impact of leg length discrepancy on clinical outcome of total hip arthroplasty: Comparison of four measurement methods. J Arthroplasty 29: 137-141.

22. Li J, McWilliams AB, Jin Z, et al. (2015) Unilateral total hip replacement patients with symptomatic leg length inequality have abnormal hip biomechanics during walking. Clin Biomech 30: 513-519.

23. Leucht P, Huddleston HG, Bellino MJ, et al. (2015) Does intraoperative fluoroscopy optimize limb length and the precision of acetabular positioning in primary THA? Orthopedics 38: e380-e386.

24. Debi R, Slamowicz E, Cohen O, et al. (2018) Acetabular cup orientation and postoperative leg length discrepancy in patients undergoing elective total hip arthroplasty via a direct anterior and anterolateral approaches. BMC musculoskeletal disorders 19: 188.

25. Matta JM, Shahrdar C, Ferguson T (2005) Single-incision anterior approach for total hip arthroplasty on an orthopaedic table. Clin Orthop 441: 115-24.

26. Bourne RB, Rorabeck CH (2002) Soft tissue balancing: the hip. J Arthroplasty 17: 17-22.

27. Friberg OE (1983) Clinical symptoms and biomechanics of lumbar spine and hip joint in leg length inequality. Spine 8: 643-651.

28. Malik A, Maheshwari A, Dorr LD (2007) Impingement with total hip replacement. J Bone Joint Surg Am 89: 1832-1842.

29. Spalding TJ (1996) Effect of femoral offset on motion and abductor muscle strength after total hip arthroplasty. J Bone Joint Surg $\mathrm{Br}$ 78: 997-998.

30. Lin TJ, Bendich I, Alex S Ha, et al. (2017) A comparison of radiographic outcomes after total hip arthroplasty between the posterior approach and direct anterior approach with intraoperative fluoroscopy. The Journal of arthroplasty 32: 616-623.

31. Pasquier G, Ducharne G, Ali ES, et al. (2010) Total hip arthroplasty offset measurement: Is CT scan the most accurate option?. Orthopaedics \& Traumatology: Surgery \& Research 96: 367-375.

DOI: $10.36959 / 453 / 532$

Copyright: (C) 2020 Smith EJ, et al. This is an open-access article distributed under the terms of the Creative Commons Attribution License, which permits unrestricted use, distribution, and reproduction in any medium, provided the original author and source are credited. 